\title{
Vertebral sclerosis in adults
}

\author{
A. S. RUSSELl, J. S. PERCY, ${ }^{1}$ AND B. C. LENTLE ${ }^{2}$ \\ 1 From the Rheumatic Disease Unit, Department of Medicine, University of Alberta, Edmonton, and the \\ ${ }^{2}$ Department of Nuclear Medicine, W. W. Cross Cancer Institute, Edmonton, Alberta
}

SUMMARY Narrowing of the intervertebral disc space with sclerosis of the adjacent vertebral bodies may occur as a consequence of infection, neoplasia, trauma, or rheumatic disease. Some patient have been described with backache and these radiological appearances without any primary cause. being apparent. The lesions were almost always of 1 or, at most, 2 vertebrae and most frequently̌ involved the inferior margin of L4. We describe 3 patients with far more extensive vertebral involve $\vec{\delta}$ ment and present the clinical, radiological, scintiscan, and histological findings. The only patiene we have seen with the better known, isolated L4/5 lesion was shown on biopsy to have staphylococcal osteomyelitis. For this reason we would still recommend a biopsy of all such sclerotic vertebra! lesions if they occur in the absence of other rheumatic disease.

Sclerosis of one or more vertebrae is a relatively nonspecific radiological finding and may reflect underlying neoplasia as well as chronic infection or possible trauma. There have been recent descriptions of sclerosis of one vertebral body occurring in association with reduction in height of the adjacent disc space (Williams et al., 1968; Martel et al., 1976). Sometimes the adjacent vertebra may be involved and lytic areas may be present adjacent to, or surrounded by, the sclerosis. These lesions are associated with pain and have been attributed to low grade infection, particularly when they are seen in children (Williams et al., 1968; Spiegel et al., 1972). Frank bacterial infection following surgical interference with intervertebral discs can produce similar radiological changes, and the term 'discitis' has been used to describe these lesions as well as those occurring in children. Similar radiological appearances may be seen in patients with ankylosing spondylitis (Cawley et al., 1972) and, more rarely, in those with rheumatoid arthritis (Seaman and Wells, 1961).

Martel et al. suggested that in adults most of these lesions are initiated by vertebral and plate fractures and are not due to a primary inflammatory lesion of the intervertebral disc, implying therefore that the term 'discitis' may be inappropriate (Martel $e l$ al., 1976; Martel, 1977). In a recent review of 17 adult patients with no other rheumatic disease the sclerotic lesions predominantly involved the inferior aspect of L4 and extended across only 1 disc space (Martel $e t$ al., 1976).

Correspondence to Dr A. S. Russell, Department of Medicine, 9-112 Clinical Sciences Building, University of Alberta, Edmonton, Alberta, Canada T6G 2G3.
We have recently seen 3 patients who have ver $\overrightarrow{0}$ tebral sclerosis and disc space narrowing but of ab more sensitive nature than has been previouslyt described. Nonmarginal syndesmophytes were also present in 1 patient. The single adult patient seen during this 2-year period which an infected vertebra lesion is also recorded for comparison.

\section{Patients and methods}

Bone scans were performed with $99 \mathrm{~m}$ technetiun stannous pyrophosphate (TcPP) as previously described (Russell et al., 1975). All showed abnormat spinal uptake. The sacroiliac/sacrum (SI/S) ratios were within normal limits except in case 2 , where the ratio was $1 \cdot 51$ and $1 \cdot 37 / 1$. None had changes outside the axial skeleton. All patients had normal sacroß iliac radiographs. All patients had a complete blood count, erythrocyte sedimentation rate, and serum calcium, phosphate, and alkaline phosphatase measured. Apart from the ESR these were all nor mal. Tests were performed for antibodies to Brus cella abortus, Francisella tularensis, salmonellae Yersinia enterocolitica selected serotypes, Yer sinia pseudotuberculosis types I, II, and III. These were negative. Mantoux tests were negative to 10d TU. HLA typing was performed, no patient pos sessed HLA B27.

\section{CASE 1}

This woman developed backache when aged $37 \overrightarrow{\mathrm{D}}$ and 3 years later a ganglioneuroma of the right $S$ 更 nerve root was removed with a partial laminectom of L5-S1. Preoperative radiological investigatio 
showed lumbar vertebral sclerosis and early syndesmophytes on the right side. Her low back symptoms improved, but 2 years later in 1975 she developed a constant pain in the mid-dorsal spine.

Examination revealed the residual signs of a right S1 nerve root lesion and a smooth dorsal kyphosis with midline tenderness from D5 to D11. Symmetrical plaques 3 to $5 \mathrm{~cm}$ across resembling nummular psoriasis were present over the limbs. Biopsy of these lesions failed to provide histological confirmation of psoriasis. Routine blood tests were all normal except for an ESR of 48. Cerebrospinal fluid (CSF) was normal. Radiological examination of the spine showed a fusion $\mathrm{C} 1$ and $\mathrm{C} 2$ of probable congenital origin. Diffuse sclerosis of D7-D10 was present, with reduction of the intervertebral disc spaces (Figs. 1 and 2). Mild but similar changes occurred in L2-L4, and in addition large right-sided

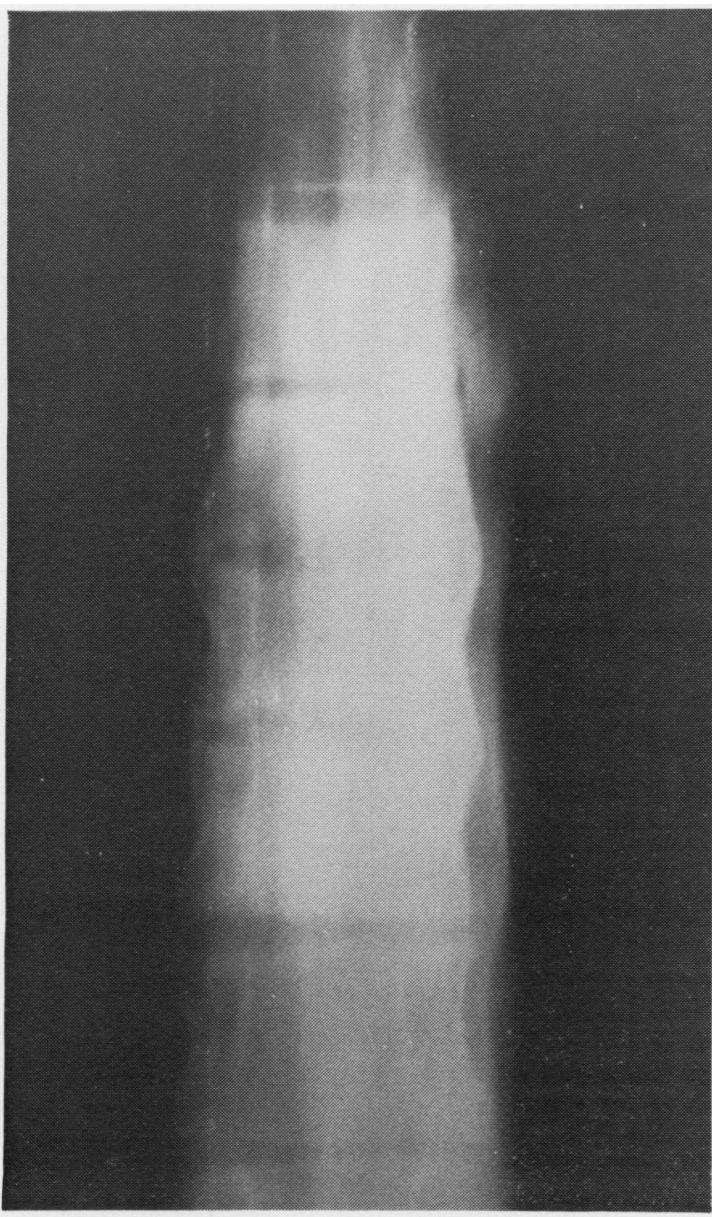

Fig. 1 Case 1. Tomograph of mid-dorsal spine.

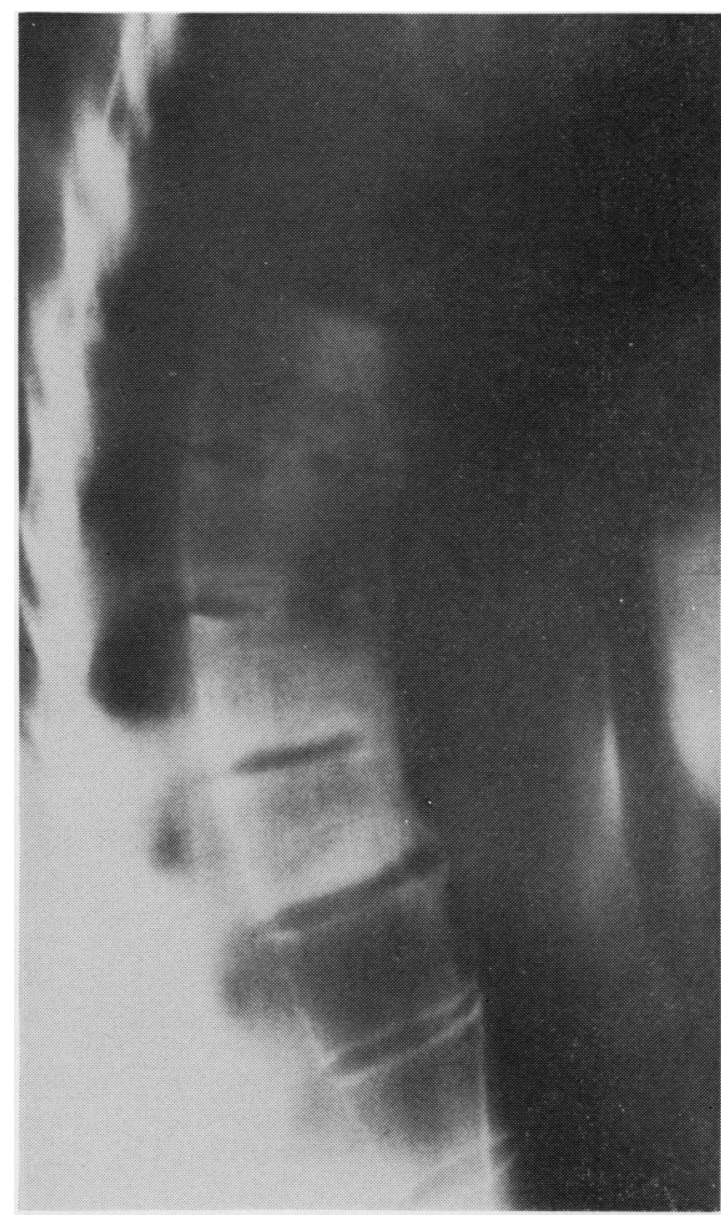

Fig. 2 Case 1. Tomograph of mid-dorsal spine.

non-marginal syndesmophytes were present (Fig. 3). A bone scan with TcPP showed increased uptake in the corresponding areas, with normal SI/S ratios.

She was treated symptomatically with indomethacin and physiotherapy and her symptoms improved, though they were not completely relieved. Her ESR 3 months later was $26 \mathrm{~mm} / \mathrm{h}$. Two years later the bone scan was normal, but radiographs were unchanged.

\section{CASE 2}

This 32-year-old farmer's wife had had a progressive mid-dorsal back pain for 5 years. Examination was normal except for midline tenderness over D6. All the blood tests were normal except for an ESR of $45 \mathrm{~mm} / \mathrm{h}$. Radiographs showed irregular sclerosis of the vertebral bodies D6/7 with reduction in the height of the intervening and adjacent discs (Fig. 4). A bone scan with TcPP showed increased uptake 


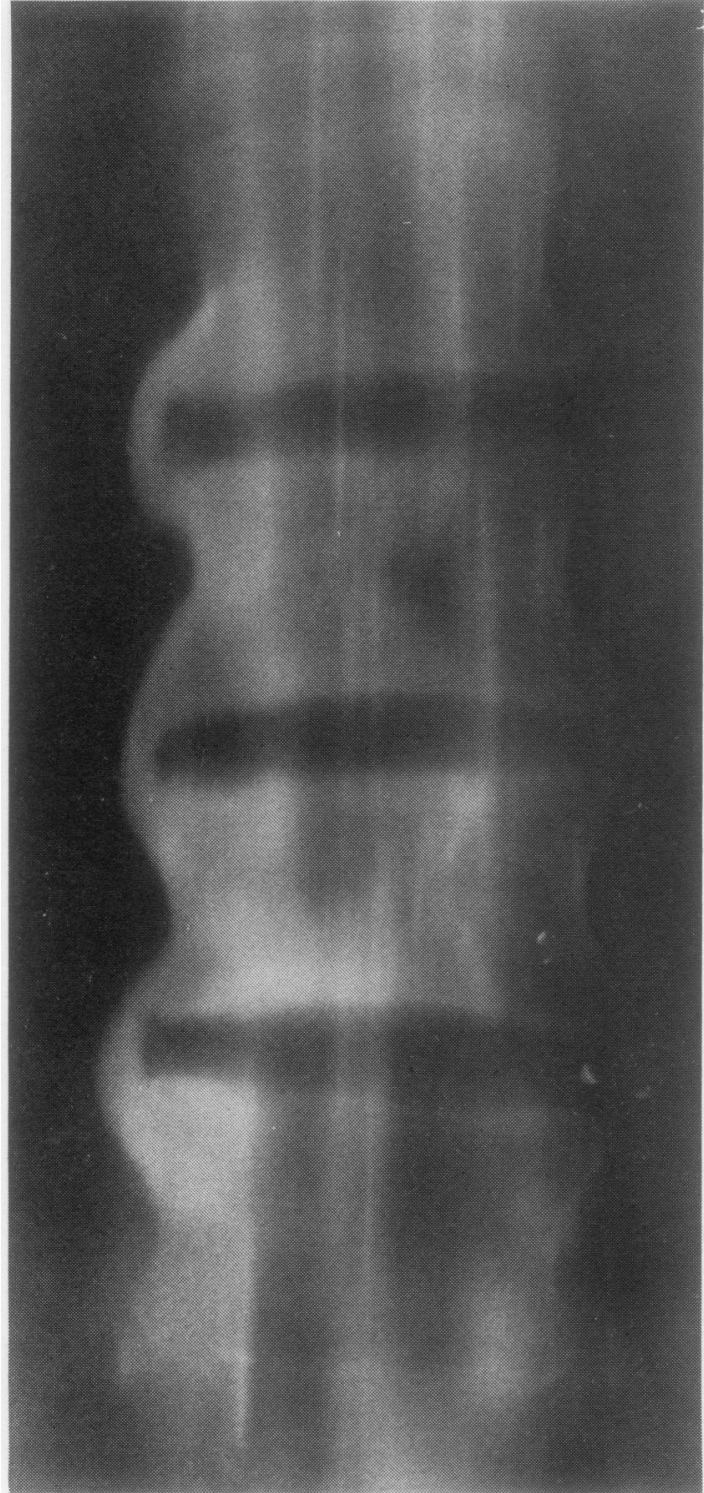

Fig. 3 Case 1. Tomograph of lumbar spine showing right-sided nonmarginal syndesmophytes.

in the mid-dorsal spine, but the sacrum/sacroiliac ratios were also increased $(1 \cdot 51 / 1 \cdot 37)$. An open biopsy of the body of D6 showed sclerotic bone associated with an increase in paravertebral fatty tissue containing small collections of histocytic cells. Aerobic and anaerobic cultures were sterile. Three months later the ESR had fallen to $22 \mathrm{~mm} / \mathrm{h}$, and both this and her radiological and scintiscan findings have remained unchanged over the ensuing 18 months. She continues to complain of some pain iọp her mid-dorsal spine, although this is less severe tha it was initially.

CASE 3

This 47-year-old man had had lumbar and dorsa backache for 4 years. It increased in severity ovee the preceding year despite the use of anti-inflames matory analgesics. He had developed pain radiating anteriorly across both sides of the chest and had increasing stiffness of the lumbar spine. There was $\overrightarrow{\mathrm{a}}$ mild tenderness over the mid-dorsal and lumbaos spine and forward flexion was restricted. Examina tion was otherwise normal. All blood tests were normal except for an ESR of $26 \mathrm{~mm} / \mathrm{h}$. Routine. radiological examination was not seen to be $a b-$ normal, but a bone scan with TcPP showed increase uptake centred over D6-D9. Tomograms showed increased bone density of these 4 vertebrae, with irregularity and narrowing of the intervening dis 8 spaces. A myelogram was normal, and the CSF was

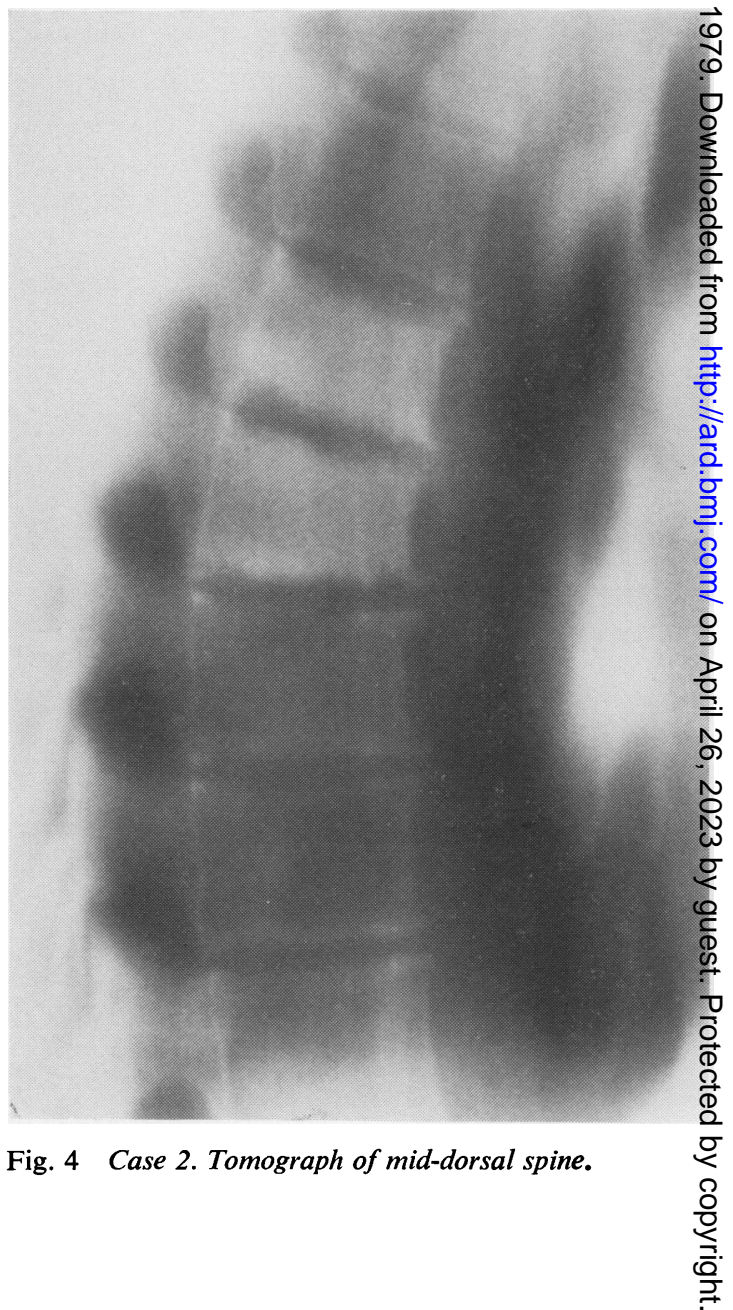


also normal. A normal biopsy was obtained from the D8 vertebra and the D8/9 disc. Symptomatic treatment was complicated by the presence of a chronic peptic ulcer. One year later his symptoms had virtually disappeared and the bone scan had returned to normal. The radiological appearances remained unchanged.

\section{CASE 4}

This 34-year-old man developed an acute low back pain while using a meat grinder. After 5 months he was put in an exercise and intermittent lumbar traction, with improvement. The pain recurred after some weeks, and he was transferred for further assessment. There was no abnormality on physical examination. Investigations showed an ESR of 41 $\mathrm{mm} / \mathrm{h}$, a globulin level of $4.9 \mathrm{~g} / 100 \mathrm{ml}$ and an IgG of $2955 \mathrm{mg} / 100 \mathrm{ml}$. All other blood tests were normal. Initial radiological examination of the lumbosacral spine with myelography had been normal but he subsequently developed vertebral sclerosis and disc space narrowing at the L4/5 level (Figure 5). A

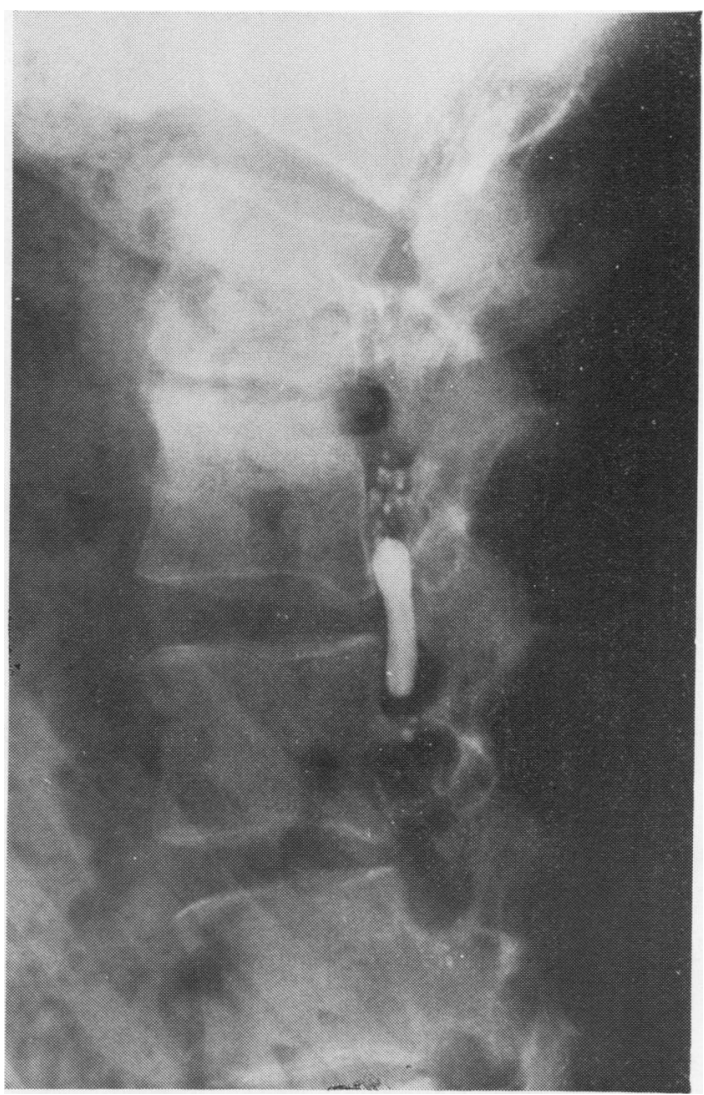

Fig. 5 Case 4. Plain radiograph of lumbar spine. repeat myelogram showed no other abnormality but the CSF obtained had $85 \mathrm{WBC}$ and $25 \mathrm{RBC} /$ $\mathrm{mm}^{3}$ and the CSF protein was mildly increased at $54 \mathrm{mg} / 100 \mathrm{ml}$. A bone scan showed increased vertebral uptake at the level of the L4/5. A biopsy of disc and bone were performed and showed osteomyelitis of both L4 and L5 with sequestrum formation. Cultures grew Staphylococcus aureus sensitive to penicillin. His symptoms resolved on long term treatment with this antibiotic.

\section{Discussion}

Vertebral sclerosis with irregularity of the vertebral end plates and reduction in height of the adjacent disc is a complication of ankylosing spondylitis (Cawley et al., 1972) and is also seen, although more rarely, in patients with rheumatoid arthritis (Seaman and Wells, 1961). We have excluded such patients from this report. Seventeen patients were recently described with the above radiological features (Martel et al., 1976). In contrast to our patients, all were aged over 40 years and all lesions involved the lumbar spine. In 12 they involved the inferior margin of $\mathrm{L} 4$, and in 4 only the single vertebra showed an abnormal radiological appearance. In young children a similar but more acute lesion may be seen, and the lower lumbar area is again the commonest site. Two adjacent vertebrae are generally involved, and there is marked reduction in height of the intervening disc space. The lesion probably has an infective cause. The only one of our patients, case 4 , with relatively restricted vertebral disease as described by Martel et al. (Martel et al., 1976; Martel, 1977) did have a lesion of L4/5 but was shown on biopsy to have a Staphylococcus aureus osteomyelitis. An infective lesion was clearly shown to be present in 7 of 15 patients recently reported with similar vertebral changes (McCain et al., 1978). It would therefore seem to be important to examine these lesions by biopsy even when seen at $L 4 / 5$. None of the other biopsies showed any specific changes. Case 2, with slight histocytic infiltration, has not shown any progression over the intervening 2 years. The 3 noninfected patients showed much more extensive involvement than previously described with, in case 1 , the formation of asymmetrical nonmarginal syndesmophytes.

The bone scan showed a focal area of increased uptake at the site of the lesions. This is of course entirely nonspecific from a diagnostic point of view but indicates that the process was associated with osteoblastic activity (Gates, 1977). In case 3 it showed an abnormality not readily apparent on initial routine radiographs but one that was visualised by tomography. We have previously reported 
the appearance of these discovertebral lesions on a bone scan in patients with ankylosing spondylitis and have shown the bone scan in this clinical situation to be abnormal before the radiological appearances of vertebral sclerosis develop (Lentle et al., 1977).

It is certainly possible that the radiological appearances we have noted are due to intervertebral disc herniation and are simply more extensive than those described by Martel et al. However, none of their patients with sclerotic lesions were under $\mathbf{4 0}$ years of age nor did any of the lesions involve the dorsal spine. We have no convincing alternative explanations, but despite the negative biopsies the raised ESR did suggest an inflammatory basis for these lesions. We would like to draw attention to these radiological appearances and to their apparently benign nature.

\section{References}

Cawley, M. I. D., Chalmers, T. M., Kellgren, J. H., and Ball, J. (1972). Destructive lesions of vertebral bodies in ankylosing spondylitis. Annals of the Rheumatic Diseases, 31, 345-358.
Gates, G. F. (1977). Scintigraphy of discitis. Clinics incs Nuclear Medicine, 2, 20-25.

Lentle, B. C., Russell, A. S., Percy, J. S., and Jackson, F. I. (1977). Scintigraphic findings in ankylosing spondylitisJournal of Nuclear Medicine, 18, 524-528.

Martel, W. (1977). A radiologically distinctive cause, of low back pain. Arthritis and Rheumatism, 20, 1014-1018.

Martel, W., Seeger, J. F., Wicks, J. D., and Washburn, R. L. (1976). Traumatic lesions of the discovertebral junction in the lumbar spine. American Journal of Roentgenology, 127, 457-464.

McCain, G. A., Ralph, E. D., Austin, T. W., Harth, M.: Bell, D. A., and Disney, T. F. (1978). Abstract. Annals of the Royal College of Physicians and Surgeons of Canada, 11, 92.

Russell, A. S., Lentle, B. C., and Percy, J. S. (1975). Investi gation of sacroiliac disease: Comparative evaluation of $\omega$ radiologic and radionuclide techniques. Journal of Rheu- $-\infty$ matology, 2, 45-51.

Seaman, W. B., and Wells, J. (1961). Destructive lesions of the $\vec{\infty}$ vertebral bodies in rheumatic disease. American Journal of Roentgenology, Radium Therapy, and Nuclear Medicine, 86, 241-250.

Spiegel, P. G., Kengla, K. W., Isaacson, A. S., and Wilson, J. C. (1972). Intervertebral disc-space inflammation ino children. Journal of Bone and Joint Surgery, 54A, 284-296.

Williams, J. L., Moller, G. A., and O'Rourke, T. L. (1968). Pseudo-infections of the intervertebral disc and adjacent vertebrae? American Journal of Roentgenology, 103, 611615. 